\title{
The width of the third ventricle associates with cognition and behaviour in motor neuron disease
}

\author{
Juan F Vázquez-Costa*a,b,c, Sara Carratalà-Boscàd,e, José I Tembl ${ }^{\mathrm{f}}$, Victoria Fornés-
} Ferrer $^{\mathrm{g}}$, Jordi Pérez-Tur ${ }^{\mathrm{h}, \mathrm{i}, \mathrm{j},}$, Luis Martí-Bonmatí ${ }^{\mathrm{d}}$, Teresa Sevilla $\mathrm{a}^{\mathrm{a}, \mathrm{b}, \mathrm{c}, \mathrm{k}}$.

${ }^{a}$ Neuromuscular Research Unit, Instituto de Investigación Sanitaria la Fe (IIS La Fe), Valencia, Spain.

${ }^{b}$ ALS Unit, Department of Neurology, Hospital Universitario y Politécnico La Fe, Valencia, Spain.

${ }^{c}$ Centro de Investigación Biomédica en Red de Enfermedades Raras (CIBERER), Spain.

${ }^{d}$ Department of Radiology and Biomedical Imaging Research Group GIBI230, Hospital

Universitario y Politécnico La Fe and Instituto de Investigación Sanitaria la Fe, Valencia, Spain

${ }^{e}$ Multiple Sclerosis and Neural Regeneration Research Group, Fundación para la Investigación La Fe, Hospital Universitario y Politécnico La Fe, Valencia, Spain.

${ }^{f}$ Neurosonology Laboratory, Department of Neurology, Hospital Universitario y Politécnico La Fe, Valencia, Spain.

${ }^{g}$ Biostatistics Unit, Instituto de Investigación Sanitaria la Fe (IIS La Fe), Valencia, Spain.

${ }^{h}$ Laboratory of Molecular Genetics, Institut de Biomedicina de València-CSIC, Valencia

${ }^{i}$ Centro de Investigación Biomédica en Red de Enfermedades Neurodegenerativas (CIBERNED), Spain. 
${ }^{j}$ Unidad mixta de Neurología y Genética, Instituto de Investigación Sanitaria la Fe (IIS La Fe), Valencia, Spain.

${ }^{k}$ Department of Medicine, University of Valencia, Valencia, Spain.

*Corresponding author: Juan Francisco Vázquez Costa MD, Department of Neurology, Hospital Universitario y Politécnico La Fe, Avenida Fernando Abril Martorell 106, 46026 Valencia, Spain; Tel. +34-961245542; juan.vazquez.neuro@gmail.com; ORCID 0000-0002$3043-7938$

Running Title: Third ventricle: a marker of cognitive impairment in MND

Wordcount: 3431 


\section{ABSTRACT}

Objectives

An enlarged width of the third ventricle (WTV) has been described in amyotrophic lateral sclerosis (ALS) patients, although its clinical meaning is unknown. The aims of this study were to evaluate the contribution of demographical, clinical and genetic factors to the WTV in different motor neuron disease (MND) phenotypes and to assess its brain structural correlates.

Materials \& methods

The WTV was measured by transcranial ultrasound in 107 MND patients (82 diagnosed with classical ALS, 16 with progressive muscular atrophy and 9 with primary lateral sclerosis) and 25 controls. Genetic analysis, and neurological and neuropsychological examinations were performed in patients. Brain volumetric analysis of MR images were obtained in 85 patients. The association of WTV with demographic, clinical, genetic and neuropsychological variables as well as with brain volumes was assessed by multivariable models.

Results

Eighteen patients were diagnosed with genetic MND and $42.3 \%$ of patients showed executive or behavioural impairment (EBI). MND patients showed larger WTV than controls. The WTV was significantly associated with age, spinal onset and the presence of EBI, but not with the genetic background, the phenotype, or disability. Greater WTV was also associated with reduced subcortical gray matter volume, but not with the cortical or the white matter volume.

Conclusions 
The enlargement of the WTV found in the different MND phenotypes is attributable to the subcortical gray matter atrophy and is associated with cognitive and behavioural impairment. Larger longitudinal studies are needed to determine its role as biomarker in MND patients with frontotemporal dementia.

Keywords: motor neuron disease; amyotrophic lateral sclerosis; progressive muscular atrophy; primary lateral sclerosis; third ventricle; ultrasound; cognitive impairment. 


\section{Introduction}

Motor neuron diseases (MND) are neurodegenerative conditions involving upper (UMN) and/or lower motor neurons (LMN). ${ }^{1}$ Three main phenotypes can be distinguished according to the degree of UMN and LMN impairment: ${ }^{1}$ classical amyotrophic lateral sclerosis (cALS), primary lateral sclerosis (PLS), and progressive muscular atrophy (PMA). Beyond motor neurons, MND patients show involvement of other brain structures in both neuroimaging and pathological studies. $^{2-5}$ These changes might be related to the presence of extra-motor features, such as cognitive or behavioural impairment in 20\% to 50\% of MND patients. ${ }^{1,5-7}$ Language and dysexecutive deficits are the most frequent cognitive findings, a poor letter fluency being the hallmark of the latter. ${ }^{6-8}$

Transcranial sonography can measure the width of the third ventricle (WTV) in an easy and reliable manner, showing a good correlation and agreement with its manual measurement in magnetic resonance (MR) imaging. ${ }^{9}$

The WTV is believed to indirectly reflect the degree of subcortical brain atrophy, particularly on adjacent structures such as thalamus. ${ }^{10}$ In this way, it has been shown useful to monitor progressive brain atrophy associated with aging, as well as for the diagnosis or monitoring of progressive supranuclear palsy (PSP) or multiple sclerosis patients. ${ }^{11-13}$ The WTV also correlates inversely with the cognitive performance in both healthy subjects and patients with multiple sclerosis, Alzheimer's disease and Parkinson's disease. ${ }^{11,14-17}$ Recently, an enlarged WTV has been described in cALS patients, ${ }^{18,19}$ although its clinical correlate is not known. Moreover, the WTV has been studied neither in other phenotypes (PMA or PLS) nor in familial MND. 
The aims of this study were: (1) to assess the differences in the WTV between controls and different MND phenotypes; (2) to evaluate the contribution of demographical, clinical and genetic factors to this biomarker and; (3) to analyse its brain structural correlates.

\section{Methods}

\section{Subjects and definitions}

Patients diagnosed with cALS, PMA or PLS were recruited and evaluated between February 2014 and November 2017 by the same neurologist (JFVC). The cALS patients met the revised El Escorial criteria of possible, probable or definitive ALS ${ }^{20}$. Patients presenting with a progressive isolated LMN impairment, affecting at least two regions, were diagnosed with PMA after appropriately excluding other LMN syndromes. ${ }^{21}$ PLS was defined as a progressive isolated UMN impairment in at least one region other than the lumbar region, lasting more than four years. ${ }^{22}$ Patients with stroke history were excluded. The hyperechogenicity of the substantia nigra was also assessed in a subgroup of these population and the results have been published elsewhere. ${ }^{23}$ For comparison purposes, a cohort of controls without neurological diseases was also recruited.

\section{Genetic MND}

MND patients were systematically asked for family history of MND or dementia. Patients were categorised as familial MND whenever they had a positive family history as previously defined, ${ }^{24}$ or as sporadic MND when not. Both sporadic and familial MND patients were screened for C9ORF72 with repeat primed PCR and expansions were confirmed with Southern blotting. Moreover, familial MND patients not carrying a C9ORF72 expansion, SOD1, TARDBP and FUS genes were subsequently analysed by Sanger sequencing. Finally, familial MND 
patients and sporadic MND patients carrying mutations were classified as genetic MND (gMND).

\section{Clinical examination}

Age, gender, date of onset of motor symptoms, region of onset (bulbar and spinal), disability (ALSFRS-R), and the degree of UMN impairment (UMN score), ${ }^{25}$ were recorded for all MND patients.

Verbal fluency (letter P) was examined in non-anarthric patients and the verbal fluency index ${ }^{8}$ was subsequently calculated. Four anarthric patients without disability in the dominant hand were examined with both the reverse digit span and the trail making test. Cut offs of each executive test were defined by the normative values, adjusted by age and education level, published in the Spanish population. ${ }^{26,27}$

Moreover, the diagnosis of behavioural frontotemporal dementia (bvFTD) was based on the Rascovsky criteria, ${ }^{28}$ after interviewing the caregiver about the presence in the patient of: a) disinhibition, b) loss of sympathy and empathy, c) perseverative, stereotyped or compulsive behaviour, d) hyper-orality/dietary change, e) apathy. Mild executive, mild behavioural impairment and bvFTD were diagnosed according to current criteria. ${ }^{8}$ These criteria require the presence of either an impaired verbal fluency or two other executive non-overlapping measures for the diagnosis of executive impairment; the identification of either apathy or two other behavioural symptoms for the diagnosis of mild behavioural impairment; and the identification of at least three behavioural Rascovsky criteria for the diagnosis of bvFTD.

A subset of patients were also assessed with the frontal assessment battery (FAB) $(n=79)$, which ranges from 0 to 18, with higher scores indicating better cognition. For the statistical analysis, the item-adjusted FAB scores were calculated as previously published ${ }^{29}$ according to 
the formula: item-adjusted score $=$ original score $* 100 / \%$ of items performed. Moreover, in some patients ( $\mathrm{n}=61$ ) the Spanish validated version of the Frontal Systems Behaviour Scale (FrSBe), ${ }^{30}$ was carried out by the caregiver. The FrSBe is composed of 46 behavioural items, each of them ranging from 1 (almost never) to 5 (almost always), which are grouped in three subscales: apathy, disinhibition, and executive dysfunction. For the present study, we have considered the Z-scores of the post-illness forms. ${ }^{30}$

\section{Transcraneal sonography examination}

An expert neurologist sonographer (JIT), blind to the clinical data, performed the ultrasound examination with the same system (Toshiba Aplio XG, Tokyo, Japan 2008), equipped with a 2.5 MHz phased-array transducer to obtain B-mode images through a temporal acoustic bone window. The WTV was obtained and measured by this examiner, as previously published. ${ }^{31}$

\section{MR imaging acquisition and processing}

MRI examinations were performed on a 3T MRI scanner (Signa HDxt, GE Healthcare, Milwaukee, USA) using a transmit-receive head coil array with 8 elements. The MR imaging protocol included a 3D T1-weighted axial fast spoiled gradient recalled sequence (TR, 6.6 ms;

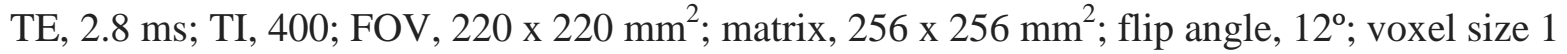
x $\left.0.94 \times 0.94 \mathrm{~mm}^{3}\right)$.

Brain volumes were automatically segmented and measured by FreeSurfer version 5.2 (http:// surfer.nmr.mgh.harvard.edu/). Segmentations were subsequently inspected and errors were corrected when present. For the purpose of this study, besides the third ventricle volume, following global measures of brain volumes provided by FreeSurfer were considered: total intracranial volume (TIV), cortex, subcortical gray matter (which includes thalamus, caudate, 
putamen, pallidum, hippocampus, amygdala, accumbens and the ventral diencephalon) and white matter.

\section{Statistical analysis}

Data were summarized using mean (standard deviation) and median ( $1^{\text {st }}$ and $3^{\text {rd }}$ quartiles) in the case of continuous variables and relative and absolute frequencies in the case of categorical variables. WTV age- and gender-controlled differences between patients and controls, and between sporadic and genetic MND patients, were assessed with a linear regression model. The association of the demographical and clinical variables of the MND patients with the WTV was analysed with linear regression models. Firstly, a group of clinical covariates (age, gender, phenotype, gMND, disease duration, ALSFRS-R, progression's rate and executive or behavioural impairment) were pre-selected based on previous literature and the study goal. Finally, the combination of covariates that best fitted the model was selected according to the Akaike Information Criteria (AIC). Since the larger brains (TIVs) associate with larger brain structures (including the third ventricle), ${ }^{32}$ we repeated the best clinical model but controlling for TIV in the 85 subjects with available MRI. Moreover, the association of brain volumes with the WTV, were also assessed with a linear regression model. The models were extended with the variable "Patient" as a random effect with random intercept in order to correct the nonindependence of the data. Pearson's correlation, with a false discover rate correction to adjust for multiple comparisons, was performed to study the relationship between WTV and cognitive and behavioural tests. Finally, the diagnostic accuracy of the WTV for cognitive impairment was analysed with the bootstrap-validated AUC, after controlling for those variables that associated with the WTV in previous models. P values $<0.05$ were considered statistically significant. All the statistical analyses and graphs were carried out using R software (version 3.4.3). 


\section{Ethical approval}

The study was approved by the Ethics Committee for Biomedical Research of the La Fe Hospital (Valencia, Spain) and has been conducted according to the principles of the Declaration of Helsinki. All the participants, or their next of kin in those incompetent ALS-FTD patients, gave written informed consent.

\section{Results}

\section{Population characteristics}

The study included 117 MND patients (91 cALS, 16 PMA and 10 PLS) and 25 controls. The demographical, clinical, genetic and ultrasound characteristics of both, patients and controls, are summarised in Table 1. Overall, MND patients were moderately disabled and the mean time from symptoms onset ranged between 14 months in cALS to 55 months in PMA patients. Eighteen patients (15.4\%) were classified as gMND. Thirteen patients could not be cognitively examined due to great motor disability. Some degree of executive or behavioural impairment (EBI) was diagnosed in 44 (42.3\%) of the remaining patients: 18 patients (17.3\%) were diagnosed with isolated mild dysexecutive impairment; 11 patients (10.6\%) with mild behavioural impairment; 9 patients (8.6\%) showed both mild dysexecutive and behavioural impairment; and 6 patients (5.8\%) with FTD.

\section{WTV in patients and controls}

Ten MND patients (8\%) showed a poor temporal acoustic bone window. Therefore, the WTV could be measured in 107 patients. MND patients showed larger WTV than the healthy controls (Estimate $=0.89[0.04,1.76], \mathrm{p}=0.042)$, after controlling for age and sex. Moreover, age 
$($ Estimate $=0.09[0.06,0.12], \mathrm{p}<0.001)$ and male sex $($ Estimate $=0.95[0.27,1.62], \mathrm{p}=0.006)$ were strongly associated with greater WTV in the whole cohort.

\section{Modifiers of the WTV in the MND patients}

Genetic MND was not associated with changes in the WTV after controlling for age and sex (Estimate $=0.93[-0.151,0.336])$ and the WTV appeared similar independently of the causal mutation (Figure 2).

In the multivariable analysis, age, male sex and EBI, but not other clinical variables (phenotype, site of onset, disease duration and ALSFRS-R), were directly associated with the WTV (Table 2). When controlling for the TIV, the effect of male sex on the WTV disappeared, and the effect of EBI and of spinal onset increased, the latter reaching the statistical significance (Table 3).

\section{WTV and cognitive impairment}

The WTV correlated with the adjusted FAB $(r=-0.68)$, the VFI $(r=0.37)$ and the dysexecutive $(\mathrm{r}=0.51)$ and apathy $(\mathrm{r}=0.48)$ subscales of the FRSBE (Figure 3).

Although MND-bvFTD patients consistently showed larger WTV than age and sex matched cognitive normal patients (Figure 4), there was considerable overlap on the WTV between all three cognitive subgroups (Figure 5). Moreover, the WTV showed limited diagnostic accuracy to predict EBI even when controlled either by age and sex (AUC 0.794) or by age, site of onset and TIV (AUC 0.776).

\section{WTV and brain volumes}

MR images were available in 85 patients within 3 months of the sonographic exam. A strong correlation between the WTV and the third ventricle volume (as per MR) was found $(r=0.887$ 
[0.83, 0.93]; Figure 6). In the multivariable analysis, after controlling for the TIV, greater WTV was associated with reduced total subcortical gray volume (Estimate $=-0.211[-0.393,-0.03], \mathrm{p}=$ 0.023), but not with the cortex (Estimate $=-0.028[-0.253,0.197], \mathrm{p}=0.806$ ) or the white matter volume (Estimate $=-0.19[-0.393,0.014], \mathrm{p}=0.067)$.

\section{Discussion}

Our study confirms previous reports ${ }^{18,19}$ of an enlarged WTV in cALS patients and demonstrates that this enlargement can be also found in other MND such as PMA and PLS, since it is not associated with the motor phenotype, but with the presence of EBI.

In healthy controls, the WTV is known to be enlarged in male subjects, due to their overall larger brain volumes, and to progressively increase with aging. ${ }^{11}$ Moreover, as a marker of brain atrophy, it is also enlarged in a great variety of neurodegenerative diseases, ${ }^{13}$ particularly in PSP patients, ${ }^{31}$ where it has been proposed as a progressions biomarker. ${ }^{33}$

Interestingly, the WTV predicts future cognitive impairment in aged controls. ${ }^{11}$ Furthermore, it also correlates with cognitive performance in multiple sclerosis, Alzheimer’s or Parkinson's disease. $^{14-17}$

Although the WTV is considered to be a marker of subcortical brain atrophy, particularly the thalamus, ${ }^{10}$ neuroimaging studies addressing the association of specific brain regions with the WTV are lacking. Therefore, the anatomic correlates and exact meaning of the WTV enlargement in ALS remain unknown.

\section{The WTV in MND}

The WTV has been previously found to be similar in cALS and Parkinson's disease patients ${ }^{34}$ but larger than in controls. ${ }^{18,19}$ Moreover, the third ventricle's volume is known to increase in 
cALS with the disease duration. ${ }^{35}$ However, the clinical correlates of this structure have been scarcely studied and the results are either controversial, ${ }^{18,19,34}$ or showing several limitations such as the lack of control by age, sex and TIV or the use of unsuitable cognitive tests (MMSE). ${ }^{18}$

In our study, the WTV was greater in MND patients than controls. As expected, the WTV was influenced by age but not by sex after controlling for TIV, confirming that males have larger WTV because they have larger brains. ${ }^{32}$ However, neither motor phenotypes nor the disease duration or disability were associate with the WTV. Conversely, the WTV was independently associated with the spinal onset, after controlling for TIV. A previous study showed larger WTV in bulbar onset patients, ${ }^{34}$ while another study failed to find differences. ${ }^{19,34}$ However, these studies did not control for co-variables (such as age, sex or cognitive impairment) that influence the WTV and are unevenly distributed in spinal and bulbar onset patients. Previous MRI studies have shown larger atrophy of motor-related gray and white matter structures in spinal onset patients even after controlling for disability, whereas extra-motor structures were more frequently impaired in bulbar onset patients, probably related with the increased frequency of cognitive impairment. ${ }^{36,37}$ Since our model controlled for cognitive impairment, the larger WTV found in spinal onset patients could actually reflect a larger atrophy in motor-related subcortical structures.

The WTV also independently associated with the EBI, an association which increased after controlling for TIV. In MND, there is a continuum of cognitive and behavioural changes, which seems to be independent of the motor phenotype. ${ }^{6,7}$ In our study, the WTV correlated with those tests assessing executive tasks and apathy, which are hallmarks of these cognitive and behavioural changes in MND patients. 
Moreover, we found that the subcortical gray matter volume, but not the cortex or the white matter volumes, were associated with the WTV. The subcortical gray matter volume comprises basal ganglia, thalamus, hippocampus and accumbens among other structures. Interestingly, atrophy of these structures has been found in ALS-FTD vs. cognitively normal ALS patients. ${ }^{6}$ Moreover, atrophy in thalamus appears to mirror the progressive frontotemporal cortical involvement in ALS patients. ${ }^{38}$ Given their anatomical localization is not surprising that the atrophy of subcortical gray matter structures results in an enlargement of the WTV. Furthermore, recent pathologic and neuroimaging studies have confirmed the presence of widespread brain atrophy and TDP-43 pathology in a subgroup of ALS patients characterized by higher rates of cognitive impairment ${ }^{3,39}$ and poor prognosis. ${ }^{40}$ Altogether, our study suggests that the WTV enlargement in some MND patients is largely attributable to the atrophy of subcortical gray matter structures involved in executive and behavioural tasks and could therefore be a marker of the extra-motor disease spread.

Despite the correlation of the WTV with cognitive and behavioural tests, the ability to predict EBI based on the WTV was limited, mainly because there was considerable overlap in the WTV between mild cognitively impaired and cognitively normal patients. The diagnostic accuracy of the WTV for the bvFTD diagnosis could not be analysed due to the low number of bvFTD-MND cases. Moreover, since only executive tests were performed in this study, the impairment in other cognitive areas might have been missed in subjects considered to be cognitive normal, limiting the specificity of the WTV. Furthermore, other non-controlled factors such as vascular risk factors could influence the WTV. A longitudinal assessment of the WTV would account for these interindividual differences, and might allow the prediction or monitoring of a progressive cognitive impairment. As a progression's biomarker would be especially 
suitable for ALS patients, since it can be easily obtained in advanced disease stages (even in patients requiring ventilation support) at bedside and does not require post-processing, unlike MRI-based biomarkers.

Finally, unlike the hyperechogenicity of the substantia nigra, ${ }^{23}$ our study was unable to find differences in sporadic vs. genetic MND patients. This result should be considered with caution due to the low number of gMND patients and the heterogeneity of the causal mutations. Indeed, different mutations cause diverse patterns of brain atrophy., ${ }^{3,41}$

\section{Strengths and limitations}

Our study represents the largest and most thorough study of the clinical meaning of the WTV in ALS patients. We included phenotypes (PMA and PLS) and variables that have not been analysed before and, by using multivariable analysis, also limit some methodological pitfalls of previous studies. Moreover, the structural correlates of the WTV were also assessed. However, our study also has some limitations. First, a larger and better characterised cohort of controls would have been desirable. However, this cohort was big enough to find statistically significant differences with the cohort of patients, after correcting for age and sex. Second, the sample size for some studied variables (genetic ALS or bvFTD-ALS) was small as their prevalence is low. Third, although the diagnosis of executive and behavioural impairment followed current

diagnostic criteria, ${ }^{8}$ a more comprehensive neuropsychological examination could have increased the accuracy of the WTV as a marker of cognitive impairment. Moreover, FrSBe has not been validated in ALS patients and behavioural changes (in particular apathy) could have been overestimated due to motor impairment. However, FrSBE is a widely used questionnaire for the behavioural examination in ALS patients that has been validated in the Spanish population, 
whereas ALS-specific questionnaires have not. Moreover, in the apathy items, relatives were specifically instructed to rate patients’ initiative beyond the motor impairment.

\section{Conclusions}

We show that the enlargement of the WTV found in MND patients is attributable to the atrophy of subcortical gray matter structures and associates to cognitive and behavioural impairment. Larger longitudinal studies are needed to determine its role as a diagnostic or prognostic biomarker, especially in ALS-bvFTD patients. 


\section{Acknowledgments}

We would like to thank patients and control subjects for their participation in this study. We also thank all neurologists who collaborated with the patient recruitment and to the anonymous reviewers, whose insightful comments helped to improve our manuscript. Samples were processed, preserved and delivered by the Biobank La Fe (PT13/0010/0026).

Conflicts of interest: The authors declare that they have no conflict of interest.

\section{Funding}

JFVC is funded by the Instituto de Salud Carlos III (ISCIII). The Centro de Investigación Biomédica en Red de Enfermedades Raras (CIBERER) and the Centro de Investigación Biomédica en Red de Enfermedades Neurodegenerativas (CIBERNED) are initiatives from the ISCIII. 


\section{REFERENCES}

1. Al-Chalabi A, Hardiman O, Kiernan MC, Chiò A, Rix-Brooks B, van den Berg LH. Amyotrophic lateral sclerosis: moving towards a new classification system. Lancet Neurol. 2016;15(11):1182-1194. doi:10.1016/S1474-4422(16)30199-5.

2. Menke RAL, Körner S, Filippini N, et al. Widespread grey matter pathology dominates the longitudinal cerebral MRI and clinical landscape of amyotrophic lateral sclerosis. Brain. 2014;137(Pt 9):2546-2555. doi:10.1093/brain/awu162.

3. Westeneng HJ, Walhout R, Straathof M, et al. Widespread structural brain involvement in ALS is not limited to the C9orf72 repeat expansion. $J$ Neurol Neurosurg Psychiatry. 2016;87(12):1354-1360. doi:10.1136/jnnp-2016-313959.

4. Braak H, Brettschneider J, Ludolph AC, Lee VM, Trojanowski JQ, Del Tredici K. Amyotrophic lateral sclerosis--a model of corticofugal axonal spread. Nat Rev Neurol. 2013;9(12):708-714. doi:10.1038/nrneurol.2013.221.

5. Agosta F, Ferraro PM, Riva N, et al. Structural brain correlates of cognitive and behavioral impairment in MND. Hum Brain Mapp. 2016;37(4):1614-1626. doi:10.1002/hbm.23124.

6. Raaphorst J, de Visser M, van Tol M-J, et al. Cognitive dysfunction in lower motor neuron disease: executive and memory deficits in progressive muscular atrophy. J Neurol Neurosurg Psychiatry. 2011;82(2):170-175. doi:10.1136/jnnp.2009.204446.

7. de Vries BS, Rustemeijer LMM, van der Kooi AJ, et al. A case series of PLS patients with frontotemporal dementia and overview of the literature. Amyotroph Lateral Scler Front Degener. 2017;18(7-8):534-548.

doi:10.1080/21678421.2017.1354996. 
8. Strong MJ, Abrahams S, Goldstein LH, et al. Amyotrophic lateral sclerosis frontotemporal spectrum disorder (ALS-FTSD): Revised diagnostic criteria. Amyotroph Lateral Scler Front Degener. 2017;18(3-4):153-174. doi:10.1080/21678421.2016.1267768.

9. Sahuquillo P, Tembl JI, Parkhutik V, Vázquez JF, Sastre I, Lago A. The study of deep brain structures by transcranial duplex sonography and imaging resonance correlation. Ultrasound Med Biol. 2013;39(2):226-232. doi:10.1016/j.ultrasmedbio.2012.09.008.

10. Minagar A, Barnett MH, Benedict RHB, et al. The thalamus and multiple sclerosis: Modern views on pathologic, imaging, and clinical aspects. Neurology. 2013;80(2):210-219. doi:10.1212/WNL.0b013e31827b910b.

11. Wollenweber FA, Schomburg R, Probst M, et al. Width of the third ventricle assessed by transcranial sonography can monitor brain atrophy in a time- and cost-effective manner - Results from a longitudinal study on 500 subjects. Psychiatry Res - Neuroimaging. 2011;191(3):212-216. doi:10.1016/j.pscychresns.2010.09.010.

12. Kallmann B-A, Sauer J, Schließer M, et al. Determination of ventricular diameters in multiple sclerosis patients with transcranial sonography (TCS). $J$ Neurol. 2004;251(1):30-34. doi:10.1007/s00415-004-0265-y.

13. Berg D, Godau J, Walter U. Transcranial sonography in movement disorders. Lancet Neurol. 2008;7(11):1044-1055. doi:S1474-4422(08)70239-4.

14. Berg D, Mäurer M, Warmuth-Metz M, Rieckmann P, Becker G. The correlation between ventricular diameter measured by transcranial sonography and clinical disability and cognitive dysfunction in patients with multiple sclerosis. Arch 
Neurol. 2000;57(9):1289-1292. doi:10.1001/archneur.57.9.1289.

15. Rodriguez MJ, Potter E, Shen Q, et al. Cognitive and structural magnetic resonance imaging features of Lewy body dementia and Alzheimer's disease. Alzheimer's Dement. 2012;8:211-218. doi:10.1016/j.jalz.2011.04.008.

16. Dalaker TO, Zivadinov R, Ramasamy DP, et al. Ventricular enlargement and mild cognitive impairment in early Parkinson’s disease. Mov Disord. 2011;26(2):297-301. doi:10.1002/mds.23443.

17. Benedict RHB, Bruce JM, Dwyer MG, et al. Neocortical atrophy, third ventricular width, and cognitive dysfunction in multiple sclerosis. Arch Neurol. 2006;63(9):1301-1306. doi:10.1001/archneur.63.9.1301.

18. Pavlovic AM, Stevic Z, Pekmezovic T, Mijajlovic M, Jovanovic Z, Lavrnic D. Increased frequency of pathologic findings on transcranial b-mode parenchymal sonography in patients with sporadic amyotrophic lateral sclerosis. Ultrasound Med Biol. 2015;41(4):982-988. doi:10.1016/j.ultrasmedbio.2014.12.005.

19. Prell T, Schenk A, Witte OW, Grosskreutz J, Gunther A. Transcranial brainstem sonography as a diagnostic tool for amyotrophic lateral sclerosis. Amyotroph Lateral Scler Front Degener. 2014;15(3-4):244-249. doi:10.3109/21678421.2014.881499.

20. Brooks BR, Miller RG, Swash M, Munsat TL. El Escorial revisited: revised criteria for the diagnosis of amyotrophic lateral sclerosis. Amyotroph Lateral Scler Other Motor Neuron Disord. 2000;1(5):293-299.

21. Visser J, de Jong JMBV, de Visser M. The history of progressive muscular atrophy: syndrome or disease? Neurology. 2008;70(9):723-727. doi:10.1212/01.wnl.0000302187.20239.93. 
22. Gordon PH, Cheng B, Katz IB, et al. The natural history of primary lateral sclerosis. Neurology. 2006;66(5):647-653.

doi:10.1212/01.wnl.0000200962.94777.71.

23. Vázquez-Costa JF, Tembl JI, Fornés-Ferrer V, et al. Genetic and constitutional factors are major contributors to substantia nigra hyperechogenicity. Sci Rep. 2017;7(1):7119. doi:10.1038/s41598-017-07835-z.

24. Byrne S, Bede P, Elamin M, et al. Proposed criteria for familial amyotrophic lateral sclerosis. Amyotroph Lateral Scler. 2011;12(3):157-159. doi:10.3109/17482968.2010.545420.

25. Vázquez-Costa JF, Mazón M, Carreres-Polo J, et al. Brain signal intensity changes as biomarkers in amyotrophic lateral sclerosis. Acta Neurol Scand. 2018;137(2):262-271. doi:10.1111/ane.12863.

26. Pena-Casanova J, Quinones-Ubeda S, Gramunt-Fombuena N, et al. Spanish Multicenter Normative Studies (NEURONORMA Project): Norms for the Stroop Color-Word Interference Test and the Tower of London-Drexel. Arch Clin Neuropsychol. 2009;24(4):413-429. doi:10.1093/arclin/acp043.

27. Peña-Casanova J, Quiñones-Úbeda S, Quintana-Aparicio M, et al. Spanish multicenter normative studies (NEURONORMA project): Norms for verbal Span, visuospatial Span, letter and number sequencing, trail making test, and symbol digit modalities test. Arch Clin Neuropsychol. 2009;24(4):321-341. doi:10.1093/arclin/acp038.

28. Rascovsky K, Hodges JR, Knopman D, et al. Sensitivity of revised diagnostic criteria for the behavioural variant of frontotemporal dementia. Brain. 2011;134(Pt 9):2456-2477. doi:10.1093/brain/awr179. 
29. Raaphorst J, Beeldman E, Jaeger B, et al. Is the Frontal Assessment Battery reliable in ALS patients? Amyotroph Lateral Scler. 2012;14(1):1-2. doi:10.3109/17482968.2012.712974.

30. Caracuel A, Verdejo-García A, Fernández-Serrano MJ, et al. Preliminary validation of the Spanish version of the Frontal Systems Behavior Scale (FrSBe) using Rasch analysis. Brain Inj. 2012;26(6):844-852. doi:10.3109/02699052.2012.655365.

31. Sastre-Bataller I, Vázquez JF, Martínez-Torres I, et al. Mesencephalic area measured by transcranial sonography in the differential diagnosis of parkinsonism. Parkinsonism Relat Disord. 2013;19(8):732-736. doi:10.1016/j.parkreldis.2013.04.010.

32. Voevodskaya O, Simmons A, Nordenskjöld R, et al. The effects of intracranial volume adjustment approaches on multiple regional MRI volumes in healthy aging and Alzheimer’s disease. Front Aging Neurosci. 2014;6:264. doi:10.3389/fnagi.2014.00264.

33. Höglinger GU, Schöpe J, Stamelou M, et al. Longitudinal magnetic resonance imaging in progressive supranuclear palsy: A new combined score for clinical trials. Mov Disord. 2017;32(6):842-852. doi:10.1002/mds.26973.

34. Fathinia P, Hermann A, Reuner U, Kassubek J, Storch A, Ludolph AC. Parkinson's disease-like midbrain hyperechogenicity is frequent in amyotrophic lateral sclerosis. J Neurol. 2013;260(2):454-457. doi:10.1007/s00415-012-66548.

35. Westeneng H-J, Verstraete E, Walhout R, et al. Subcortical structures in amyotrophic lateral sclerosis. Neurobiol Aging. 2015;36(2):1075-1082. 
doi:10.1016/j.neurobiolaging.2014.09.002.

36. Kim H-J, de Leon M, Wang X, et al. Relationship between Clinical Parameters and Brain Structure in Sporadic Amyotrophic Lateral Sclerosis Patients According to Onset Type: A Voxel-Based Morphometric Study. PLoS One. 2017;12(1):e0168424. doi:10.1371/journal.pone.0168424.

37. Van Der Graaff MM, Sage CA, Caan MWA, et al. Upper and extra-motoneuron involvement in early motoneuron disease: A diffusion tensor imaging study. Brain. 2011;134(4):1211-1228. doi:10.1093/brain/awr016.

38. Tu S, Menke RAL, Talbot K, Kiernan MC, Turner MR. Regional thalamic MRI as a marker of widespread cortical pathology and progressive frontotemporal involvement in amyotrophic lateral sclerosis. J Neurol Neurosurg Psychiatry. 2018:in press. doi:10.1136/jnnp-2018-318625.

39. Cykowski MD, Takei H, Schulz PE, Appel SH, Powell SZ. TDP-43 pathology in the basal forebrain and hypothalamus of patients with amyotrophic lateral sclerosis. Acta Neuropathol Commun. 2014;2(1):171. doi:10.1186/s40478-0140171-1.

40. Senda J, Atsuta N, Watanabe H, et al. Structural MRI correlates of amyotrophic lateral sclerosis progression. J Neurol Neurosurg Psychiatry. 2017;88(11):901907. doi:10.1136/jnnp-2016-314337.

41. Agosta F, Spinelli EG, Marjanovic I V., et al. Unraveling ALS due to SOD1 mutation through the combination of brain and cervical cord MRI. Neurology. 2018: in press. doi:10.1212/WNL.0000000000005002. 
Figure 1. Diagram representing the flow of participants. Ten patients were excluded from the final analysis because of poor acoustic bone window. cALS: classical amyotrophic lateral sclerosis; MND: motor neuron disease; PLS: primary lateral sclerosis; PMA: progressive muscular atrophy; WTV: width of the third ventricle.

Figure 2. WTV in MND patients harbouring mutations (“C9ORF72”, "SOD1” and “FUS”), familial ALS without known mutations (“Unknown”) and sporadic patients not carrying mutations (“NO”). WTV: width of the third ventricle.

Figure 3. Correlation of the WTV with the results of executive (A and B) and behavioural tests (C-F). FRSBE values correspond to Z-scores. FAB: frontal assessment battery; FRSBE: frontal systems behavioural scale; VFI: verbal fluency index; WTV: width of the third ventricle.

Figure 4. WTV in two cALS patients and one control matched by sex (all male) and age: 67 years old control (A); 65 years old cognitive normal cALS patient (B); 66 years old cALS-bvFTD patient (C). The WTV is considerably larger in the cALS-bvFTD patient. cALS: classical amyotrophic lateral sclerosis; bvFTD: behavioural frontotemporal dementia; WTV: width of the third ventricle.

Figure 5. WTV in MND patients according to their cognitive and behavioural classification. MEBI: mild executive or behavioural impairment; WTV: width of the third ventricle.

Figure 6. Correlation between the third ventricle volume as per MRI and the WTV as per transcranial sonography. TCS: transcranial sonography; WTV: width of the third ventricle. 